\title{
Formation of single and multiple deformation twins in nanocrystalline fcc metals
}

\author{
Y.T. Zhu ${ }^{\mathrm{a}, *}$, J. Narayan ${ }^{\mathrm{a}, *}$, J.P. Hirth ${ }^{\mathrm{b}, * 1}$, S. Mahajan ${ }^{\text {c }}$, X.L. Wu ${ }^{\mathrm{d}}$, X.Z. Liao ${ }^{\text {e }}$ \\ ${ }^{a}$ Department of Materials Science and Engineering, North Carolina State University, Raleigh, NC 27695, USA \\ ${ }^{\mathrm{b}}$ (RTD) School of Mechanical \& Materials Engineering, Washington State University, Pullman, WA 99164, USA \\ ${ }^{\mathrm{c}}$ School of Materials, Arizona State University, Tempe, AZ 85287, USA \\ ${ }^{\mathrm{d}}$ State Key Laboratory of Nonlinear Mechanics, Institute of Mechanics, Chinese Academy of Sciences, Beijing 100080, China \\ ${ }^{\mathrm{e}}$ School of Aerospace, Mechanical and Mechatronic Engineering, The University of Sydney, NSW 2006, Australia
}

Received 4 March 2009; received in revised form 13 April 2009; accepted 15 April 2009

Available online 23 May 2009

\begin{abstract}
Deformation twins are often observed to meet each other to form multi-fold twins in nanostructured face-centered cubic (fcc) metals. Here we propose two types of mechanism for the nucleation and growth of four different single and multiple twins. These mechanisms provide continuous generation of twinning partials for the growth of the twins after nucleation. A relatively high stress or high strain rate is needed to activate these mechanisms, making them more prevalent in nanocrystalline materials than in their coarse-grained counterparts. Experimental observations that support the proposed mechanisms are presented.
\end{abstract}

(C) 2009 Acta Materialia Inc. Published by Elsevier Ltd. All rights reserved.

Keywords: Nanocrystalline materials; Dislocations; Twin boundaries; Twinning; Interfaces

\section{Introduction}

Twinning is one of the major deformation modes in metals and alloys [1,2]. Face-centered cubic (fcc) metals and alloys may deform by twinning under one, or a combination of several, of the following material and deformation conditions [1-8]: (i) low stacking fault energy; (ii) high strain rate; (iii) low deformation temperature and (iv) nanocrystalline grain size. Significantly, nanocrystalline metals with medium-to-high stacking fault energy have been observed to deform by twinning at normal strain rates and room temperature, although they do not deform by twinning in their coarse-grained counterparts under the same conditions [3-7]. In addition, contrary to coarse-

\footnotetext{
* Corresponding authors. Tel.: +1 919 5130559; fax: +1 9195153419.

E-mail addresses: ytzhu@ncsu.edu (Y.T. Zhu), J_Narayan@ncsu.edu (J. Narayan),jphmdh1@cox.net (J.P. Hirth).

${ }^{1}$ Present address: 114 E. Ramsey Canyon Rd., Hereford, AZ 85615,
}

grained metals, which become more difficult to deform by twinning with decreasing grain size, nanocrystalline fcc metals may be easier to deform by twinning with decreasing grain size, reaching a maximum twinning probability and then become more difficult again when the grain size decreases further, i.e. an inverse grain-size effect on twinning [9].

Deformation twins usually form in nanocrystalline fcc metals via mechanisms different from those proposed for coarse-grained materials [2]. Several twinning mechanisms have been proposed for and observed in nanocrystalline fcc metals, including the coincidental overlapping of wide stacking fault ribbons inside nanosized grains [10,11], emissions of partial dislocations from grain boundaries [2,5,6,10-17], grain boundary splitting and migration $[11,12,15]$, and random activation of partials (RAP) from grain boundaries [2]. These reports mostly concern single twins. Multiple twins, where two or more twins meet in a confined area, have also been reported. For example, fivefold deformation twins were reported in nanocrystalline $\mathrm{Al}$ 
and $\mathrm{Cu}[18-20]$. They were proposed to form by the mechanism of partial dislocation-mediated sequential twinning [19], which was also verified by molecular dynamic simulations [21]. However, this mechanism requires a strict twinning sequence to produce fivefold twins, which explains why fivefold twins are not observed very often. This mechanism cannot explain the many other multiple twins observed in nanocrystalline fcc metals.

One of the major issues in the formation of deformation twins in nanocrystalline fcc metals is the source of partial dislocations that are needed on consecutive $\left\{\begin{array}{llll}1 & 1 & 1\end{array}\right\}$ slip planes. In coarse-grained fcc metals, several continuous partial-supply mechanisms have been proposed, including the pole [22], prismatic glide [23], faulted dipole [24] and others [25-27]. However, these mechanisms cannot operate in nanocrystalline metals due to the lack of dislocation sources in the grain interior. The recently proposed RAP mechanism [2] provides for the continuous supply of partials with different Burgers vectors to grow deformation twins without macroscopic strain, but cannot explain conventional twins with such strain. Another recently proposed mechanism allows continuous self-thickening of a type of double twin observed in coarse-grained $\mathrm{Cu}-\mathrm{Ge}$ alloys [28]. A salient feature of this proposal is that, once the first Shockley partial is emitted from a grain boundary and cross slips onto another $\left\{\begin{array}{lll}1 & 1 & 1\end{array}\right\}$ plane, a deformation twin could nucleate and grow in both the primary and cross-slip planes without requiring the nucleation of additional twinning partials from the grain boundary. This mechanism has been found to operate in nanocrystalline $\mathrm{Ni}$ [29]. These two mechanisms for continuous partial generation to grow twins provide explanations for the growth of two differently configured deformation twins.

A dislocation "rebound mechanism" has been observed to occur during the deformation of thin films [30] and confirmed by molecular dynamics simulations [31], although experimental observations have suggested another mechanism at play in forming the same dislocation structure [32]. In the dislocation rebound mechanism, a dislocation impinging on a grain boundary or twin boundary at high velocity will have its elastic field reflected from the grain boundary or twin boundary. If such a mechanism operates in nanocrystalline materials, this reversed shear stress field could help nucleate a partial with an opposite sign on the next slip plane, which will glide back to grow the twin. More details will be described in Section 3.

Multiple twins with various morphologies have been frequently observed in nanocrystalline $\mathrm{Cu}, \mathrm{Al}, \mathrm{Ni}, \mathrm{Pd}$ and other fcc metals and their alloys [3-5,9,10,12,33]. However, their nucleation and growth mechanisms are not clear. Specifically, the following questions need to be answered regarding their formation: (i) How does a multiple twin nucleate? (ii) What dislocation reactions occur at the interface of multiple twins? and (iii) What is the source of the partial dislocations that cause the multiple twins to grow? In other words, are they emitted from the grain boundaries individually or are there other mechanisms for partial dislocation generation in consecutive slip planes?

In this paper, we propose two types of twinning mechanism in nanocrystalline fcc metals as classified by the sources of the twinning partials. Both types of twinning mechanism can explain the nucleation and growth of the four different single and multiple deformation twins explored in this paper.

The first type of twinning mechanism is called a self partial-multiplication twinning mechanism because the source of the twinning partials is the growing twin itself. In this mechanism, the first partials involved in the nucleation of a twin can originate from grain boundaries or other sources. Partial dislocation emissions from grain boundaries have been observed both in molecular dynamic simulations [6,7] and under high-resolution transmission electron microscopy $[3,5]$. However, after the nucleation, continuous growth of the single or multiple twins can be sustained through dislocation reactions at the growing twin interfaces or boundaries, which generates the additional twinning partials needed for twin growth.

The second type of twinning mechanism is a rebound mechanism, in which the twinning partials are generated by the rebounding of partials at a grain boundary or twin boundary. Details of these twinning mechanisms are described in the following sections.

\section{Self partial-multiplication twinning mechanisms}

Before starting to describe the four self partial-multiplication twinning mechanisms, we first describe some fundamentals of the dislocation slip systems in fcc metals and how the twinning partials in fcc metals grow a deformation twin. There are four equivalent $\left\{\begin{array}{lll}1 & 1 & 1\end{array}\right\}$ slip planes in an fcc metal: their geometric relationships are illustrated by the Thompson tetrahedron (see Fig. 1a). The angle between any two slip planes is $70.53^{\circ}$. Each edge of the tetrahedron represents the Burgers vector of a full dislocation, $\frac{1}{2}\left\langle\begin{array}{llll}1 & 1 & 0\end{array}\right)$. Unfolding the Thompson tetrahedron onto a flat surface yields a two-dimensional representation (see Fig. 1b) that is easier to read. As shown in Fig. 1b, the four $\left\{\begin{array}{lll}1 & 1 & 1\end{array}\right\}$ slip planes (represented by four triangles) can be denoted as (a),
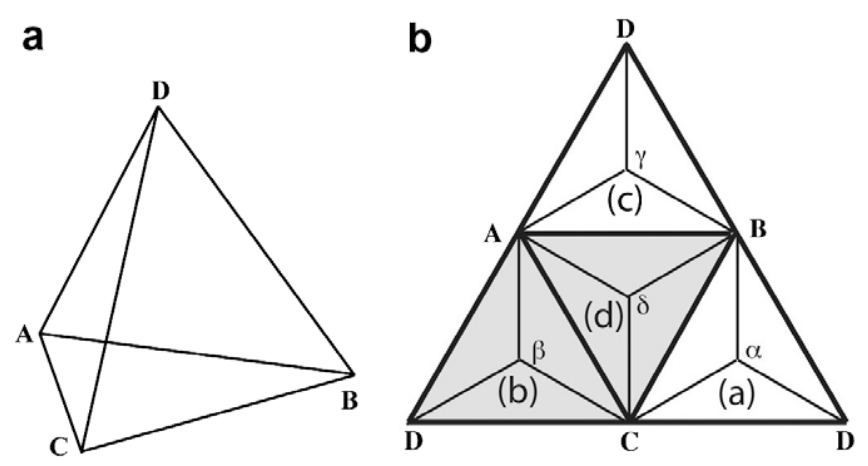

Fig. 1. (a) The Thompson tetrahedron. (b) Its two-dimensional representation. 
(b), (c) and (d). The (b) and (d) planes are shaded here because they will be used to help describe multiple deformation twins in the following sections. Note also that points $\alpha, \beta, \gamma$ and $\delta$ are at the center of the triangles $\mathrm{BCD}, \mathrm{ACD}, \mathrm{ABD}$, and $\mathrm{ABC}$, respectively. The lines that link the center of a triangle with its corners, e.g. A $\boldsymbol{\beta}$, and $\boldsymbol{\delta} \mathbf{C}$, represent the Burgers vectors of partial dislocations on that slip plane. For simplicity, we define the direction of the Burgers vector as from the first letter to the second letter. For example, $\mathbf{A} \boldsymbol{\beta}$ represents a vector from $\mathrm{A}$ to $\beta$, and $\mathbf{A C}$ from $\mathrm{A}$ to $\mathrm{C}$. A dislocation whose Burgers vector links the centers of two slip planes is defined as a stair-rod dislocation (e.g. $\beta \delta$ ).

We assume that the multiple deformation twins are formed by glide of Shockley partials on planes (d) and (b). Orienting the Thompson tetrahedron (Fig. 1a) to make AC perpendicular to the page, the (d) and (b) planes can be illustrated as in Fig. 2a. Using the axiom from Hirth and Lothe [34] (see Fig. 2b), a twin on the (d) plane can grow on the top twin boundary by the glide of the $\mathbf{C} \boldsymbol{\delta}$ (or $\mathbf{B} \boldsymbol{\delta}$ or $\mathbf{A} \boldsymbol{\delta}$ ) partial to the right or the $\boldsymbol{\delta} \mathbf{C}$ (or $\boldsymbol{\delta} \mathbf{B}$ or $\boldsymbol{\delta} \mathbf{A}$ ) partial to the left. Due to the crystal symmetry, the twin grows on the bottom twin boundary by the glide of partials whose Burgers vectors are opposite to those on the top twin boundary, as illustrated in Fig. $2 \mathrm{~b}$. Note that $T$ represents the twinned region while $M$ represents the matrix region. The thick black lines represent the twin boundary and the thin hairlines represent the slip plane. The steps in the thick black lines represent the locations of partial dislocations. We also use symbol "L" to represent the partial dislocation, and the arrow represents glide direction of the partial. These representations will be also used hereafter. The generation of the Shockley partials for the twin growth will be addressed in following sections. Using the same logic, we illustrate twin growth on (b) plane in Fig. 2c.

With the help of the Thompson tetrahedron in Fig. 1 and the configuration of partial slip for twin growth in Fig. 2, we describe the four twin growth mechanisms in the following sections.

\subsection{Growth of single twin via partial reactions at twin boundary}

This mechanism provides for a way to nucleate and grow a single twin without the requirement of an additional dislocation source from grain boundaries or another partial multiplication source in the grain interior. As shown in Fig. 3a, there is a stacking fault, SF, on the (d) plane. A partial dislocation, $\mathbf{C} \boldsymbol{\beta}$, glides on the (b) plane toward the stacking fault and is stopped on the slip plane next to the stacking fault.

Driven by applied stress, the partial $\mathbf{C} \boldsymbol{\beta}$ dissociates into a partial dislocation $\mathbf{C} \boldsymbol{\delta}$ on the (d) plane and a stair rod dislocation $\boldsymbol{\delta} \boldsymbol{\beta}$ (see Figs. $1 \mathrm{~b}$ and $3 \mathrm{~b}$ ), i.e.

$\mathbf{C} \boldsymbol{\beta} \rightarrow \mathbf{C} \boldsymbol{\delta}+\boldsymbol{\delta} \boldsymbol{\beta}$

According to Fig. $2 b, \mathbf{C} \boldsymbol{\delta}$ glides to the right to nucleate the twin, leaving behind the stair-rod dislocation $\delta \beta$, which produces the scenario shown in Fig. 3b. Note that under high applied stresses the partial $\mathbf{C} \boldsymbol{\beta}$ can also penetrate the stacking fault instead of dissociation according to Eq. (1). This scenario will be discussed later.

The stair-rod dislocation $\boldsymbol{\delta} \boldsymbol{\beta}$ can further dissociate into a partial $\delta \mathbf{C}$ on the (d) plane and a partial $\mathbf{C} \boldsymbol{\beta}$ on the (b) plane according to Fig. 1b:

$\delta \boldsymbol{\beta} \rightarrow \boldsymbol{\delta} \mathbf{C}+\mathbf{C} \boldsymbol{\beta}$

This reaction, and others to follow, would be energetically unfavorable in the absence of stress, but can become favorable under the characteristically high stresses associated with twin formation. According to Fig. $2 b, \delta \mathbf{C}$ slips to the left to extend the twin nucleus and the partial $\mathbf{C} \boldsymbol{\beta}$ glides up by one atomic plane, as shown in Fig. 3c.

The above dislocation reactions at the stacking fault $\mathrm{SF}$ nucleate a two-layer twin and leave behind the original partial dislocation $\mathbf{C} \boldsymbol{\beta}$ at the twin boundary. The partial $\mathbf{C} \boldsymbol{\beta}$ can continue the above dislocation reactions and slips as described in Eqs. (1) and (2) and illustrated in Fig. 3b and $\mathrm{c}$, with each reaction cycle growing the twin by one slip plane.
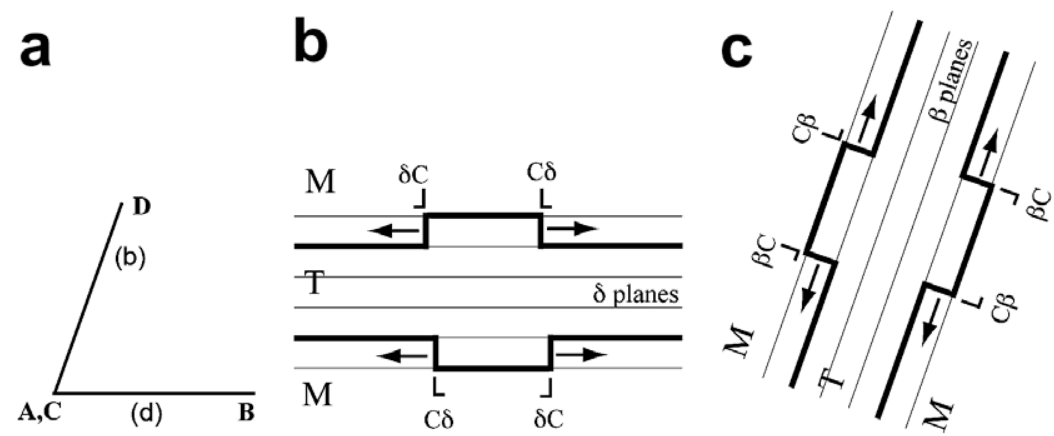

Fig. 2. (a) Projection of the (b) and (d) planes along the AC direction of the Thompson tetrahedron. (b) Growth of a twin on the (d) planes via the slips of partials $\mathbf{C} \boldsymbol{\delta}$ and $\boldsymbol{\delta} \mathbf{C}$. Note that $\mathbf{C} \boldsymbol{\delta}$ can be replaced by $\mathbf{A} \boldsymbol{\delta}$ or $\mathbf{B} \boldsymbol{\delta}$, and $\boldsymbol{\delta} \boldsymbol{C}$ can be replaced by $\boldsymbol{\delta} \mathbf{A}$ or $\boldsymbol{\delta} \mathbf{B}$. (c) Growth of a twin on the (b) planes via the glide of partials $\mathbf{C} \boldsymbol{\beta}$ and $\boldsymbol{\beta} \mathbf{C}$. Note that $\mathbf{C} \boldsymbol{\beta}$ can be replaced by $\mathbf{A} \boldsymbol{\beta}$ or $\mathbf{D} \boldsymbol{\beta}$, and $\boldsymbol{\beta C}$ can be replaced by $\boldsymbol{\beta A}$ or $\boldsymbol{\beta D}$ (see the (b) plane in Fig. 1b). The symbol "L" represents the partial dislocation, and the arrow represents glide direction. The step in (b) and (c) represents the position of the partial dislocation. The thick lines in (b) and (c) represent the twin boundaries, and the thin hairlines represent the slip planes. The sense vector $\xi$ points into the page. 


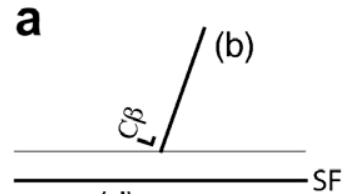

(d)

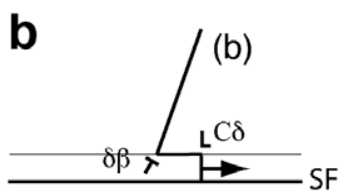

(d)

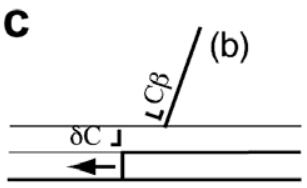

(d)

Fig. 3. Schematics of reactions between a partial, $\mathbf{C} \boldsymbol{\beta}$, on the (b) plane and a stacking fault, SF, on the (d) plane to nucleate a single twin. Repetition of these reactions leads to the growth (thickening) of this twin.

\subsection{Formation of a V-shaped double twin}

This mechanism is similar to the self-thickening crossslip mechanism we have proposed before [28,29], except that a stacking fault is involved at its activation. Note that the self-thickening cross-slip mechanism is different from the cross-slip mechanism proposed by Fujita and Mori [35]. The latter dose not provide for a mechanism for the twin to grow (thicken) by itself. We start with the situation described in Fig. 3a, in which the partial $\mathbf{C} \boldsymbol{\beta}$ is stopped at the stacking fault SF. The partial $\mathbf{C} \boldsymbol{\beta}$ now dissociates into the following dislocations (see Fig. 1b) under the applied stress:

$\mathbf{C} \boldsymbol{\beta} \rightarrow \mathbf{C A}+\mathbf{A} \boldsymbol{\delta}+\boldsymbol{\delta} \boldsymbol{\beta}$

A $\delta$ glides to the right to nucleate a twin on the (d) plane, while CA cross-slips to the next intersection of the (d) and (b) planes (see Fig. 4a). The stair-rod dislocation $\boldsymbol{\delta} \boldsymbol{\beta}$ is sessile and cannot move. Note that the dislocation reactions in Eqs. (1)-(3) are energetically unfavorable and can only proceed with the help of applied stress.

CA now dissociates into two partials on the (b) plane:

$\mathbf{C A} \rightarrow \mathbf{C \beta}+\boldsymbol{\beta A}$

BA glides upward to nucleate a twin on the (b) plane, forming the nucleus of a V-shaped double twin (see Fig. 4b).

The partial $\mathbf{C} \boldsymbol{\beta}$ can repeat the above dislocation reactions and processes, causing growth (thickening) of the double twin, as illustrated in Fig. 4c. As shown, there is a stairrod dislocation $\boldsymbol{\delta} \boldsymbol{\beta}$ at every intersection of the (d) and (b) planes. This results in a high stress at the ends of the stair-rod pileup at the intersecting boundary of the two twins. When the stress is very high, partial or perfect dislocations could be emitted or absorbed to relieve these stress concentrations. If these were partials, added stacking faults would be formed in the double twin or the adjacent matrix.

\subsection{Formation of a T-shaped twin}

A T-shaped multiple twin can be formed in a process similar to the V-shaped twin shown in Fig. 4. For simplicity, we start with a V-shaped twin as shown in Fig. 4c. We assume that a partial dislocation $\mathbf{C} \boldsymbol{\delta}$, emitted from the grain boundary or another source, glides to the right on a (d) plane adjacent to the original stacking fault (see Fig. 5a). The partial $\mathbf{C} \boldsymbol{\delta}$ is stopped by the twin boundary and dissociates into the following dislocations (see Fig. 1b) under the applied stress:

$\mathbf{C} \boldsymbol{\delta} \rightarrow \mathbf{C A}+\mathbf{A \beta}+\boldsymbol{\beta} \boldsymbol{\delta}$

A $\boldsymbol{\beta}$ glides upward to nucleate a twin on the (b) plane, while CA cross-slip to the intersection of the next (d) plane and (b) plane (similar to that shown in Fig. 4a). The stair-rod dislocation $\boldsymbol{\beta} \boldsymbol{\delta}$ is sessile and cannot move. CA now dissociates into two partials on the (d) plane:

$\mathbf{C A} \rightarrow \mathbf{C} \boldsymbol{\delta}+\boldsymbol{\delta} \mathbf{A}$

$\boldsymbol{\delta} \mathbf{A}$ glides to the left to grow the twin on the (d) plane (see Fig. 5b). The partial $\mathbf{C} \boldsymbol{\delta}$ can repeat the above dislocation reactions and processes, causing growth (thickening) of the twin, as illustrated in Fig. 5c.

As shown in Fig. 5c, the above process produces a stairrod dislocation $\boldsymbol{\beta} \boldsymbol{\delta}$ at every intersection of (d) and (b) planes that is of the opposite sign to the $\boldsymbol{\delta} \boldsymbol{\beta}$ stair-rod dislocations formed earlier in the double twin. The arrays of $\boldsymbol{\beta} \boldsymbol{\delta}$ and $\boldsymbol{\delta} \boldsymbol{\beta}$ stair-rod dislocations partially cancel each other's long-range stress fields [36]. However, the pileup tips would still have local stress concentrations.

The coherent twin/matrix boundaries are $\Sigma 3$ boundaries, while the twin-twin interfaces correspond to $\Sigma 9$ boundaries. Arrays of $\boldsymbol{\beta} \boldsymbol{\delta}$ and $\boldsymbol{\delta} \boldsymbol{\beta}$ stair-rods correspond physically to the $\Sigma 9$ boundary in two configurations.

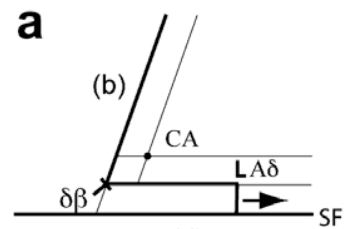

(d)

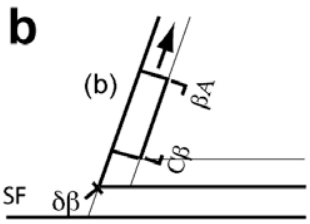

(d)

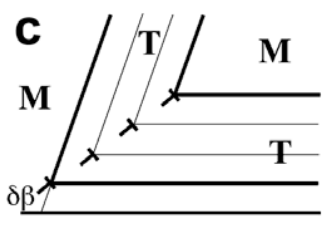

M

Fig. 4. Schematics of the nucleation and growth of a V-shaped double deformation twin. (a) Nucleation of a twin on the (d) plane. (b) Nucleation of the second twin on the (b) plane. (c) A V-shaped double twin after some growth. A stair-rod dislocation $\boldsymbol{\delta} \boldsymbol{\beta}$ is at every intersection between the (d) and (b) planes. 

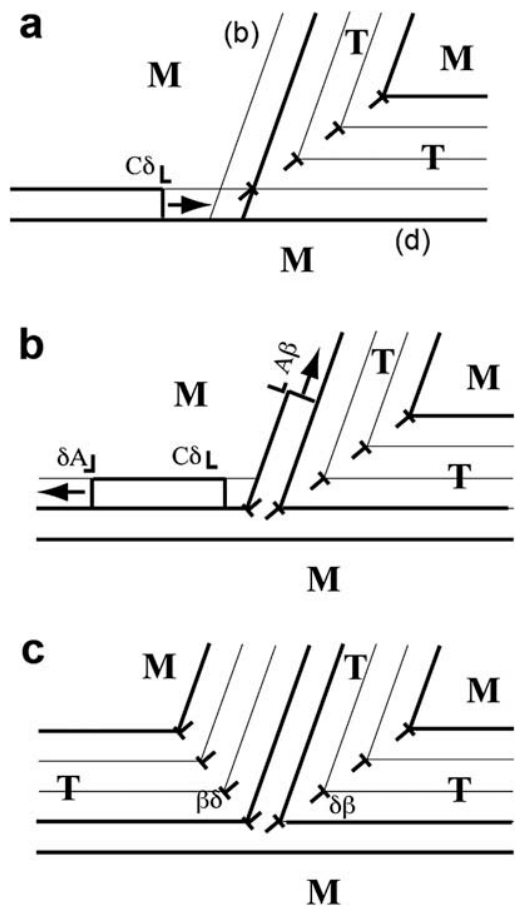

Fig. 5. Formation of a T-shaped multiple twin. (a) A partial, $\mathbf{C} \boldsymbol{\delta}$, slip toward the twin boundary. (b) After the dislocation reactions (Eqs. (5) and (6)), a T-shaped twin is nucleated, leaving behind the partial $\mathbf{C} \boldsymbol{\delta}$ to repeat another dislocation reaction cycle. (c) The final T-shaped twin with two arrays of stair-rod dislocations.

Stair-rods are "super sessile" dislocations that cannot glide or climb directly.

\subsection{Formation of an $X$-shaped twin}

To describe the formation of the X-shaped multiple twins, we start with the configuration in Fig. 3a. If the applied stress has the appropriate orientation and is high enough, the partial $\mathbf{C} \boldsymbol{\beta}$ can cut through the stacking fault and form the stacking fault configuration shown in Fig. 6a. Twins can nucleate and grow at all four reentrant angles by the mechanisms described in Sections 2.2 and 2.3, forming the X-shaped twin shown in Fig. $6 b$.

As shown in Fig. 6b, the stair-rod dislocation pileups are all in dipole arrangements. As a result, no long-range stress fields exist, but local stresses still exist at the ends of the pileups. This is energetically the most favorable configuration.
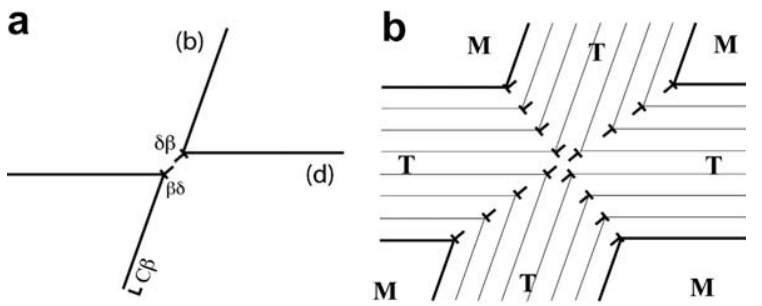

Fig. 6. The formation of an X-shaped multiple twin. (a) A partial $\mathbf{C} \boldsymbol{\beta}$ on the (b) plane cuts through a stacking fault on the (d) plane, forming four reentrant angles separated by stacking faults. (b) The final configuration of the X-shaped twin.

\section{Dislocation rebound twinning mechanisms}

The above four single and multiple twins could also be formed by the dislocation rebound mechanism [30]. The elastic field of a partial dislocation is reflected (or partially reflected) at grain boundaries or twin boundaries (analogous to shock wave reflection) [30]. The reverse shear field can aid in nucleation of an opposite-sign dislocation where the first one impinged, especially if the velocity of the dislocation approaches either the Rayleigh velocity or the transverse sound velocity $[30,31]$. This dislocation rebound mechanism can produce the single twin, $\mathrm{V}$-shaped double twin, $\mathrm{T}$-shaped multiple twin and $\mathrm{X}$-shaped multiple twin described in Sections 2.1-2.4. The rebound mechanism is deliberated in the following sections.

\subsection{Formation of a single twin by the rebound mechanism}

As shown in Fig. 7a, a partial, $\mathbf{C} \boldsymbol{\delta}$, nucleates from the grain boundary on the left and glides to the right to form a stacking fault. Such events have been observed both in molecular dynamic simulations [6,7] and under high-resolution transmission electron microscopy [5]. The elastic field of the partial dislocation is reflected by the grain boundary on the right, which helps with the nucleation of a partial dislocation with an opposite sign, $\delta \mathbf{C}$, on the next slip plane. This partial glides to the left, nucleating a single twin, as shown in Fig. 7b. Note that the nucleation and glide of $\delta \mathbf{C}$ leaves behind a partial of opposite sign, $\mathbf{C} \boldsymbol{\delta}$, on the right grain boundary (see Fig. 7b).

In a similar process, the dislocation $\delta \mathbf{C}$ could be reflected to form a partial $\mathbf{C} \boldsymbol{\delta}$ on the left grain boundary. Repetition of the process causes growth of the single twin. The twin produces a macroscopic shear and changes the shape of the grain, similar to the twins formed by monotonic activation of partials (MAP) [2].

\subsection{Formation of $V$-, $T$ - or $X$-shaped multiple twins by the rebound mechanism}

In the self partial-multiplication twinning mechanisms described in Section 2, the dislocation reaction involves

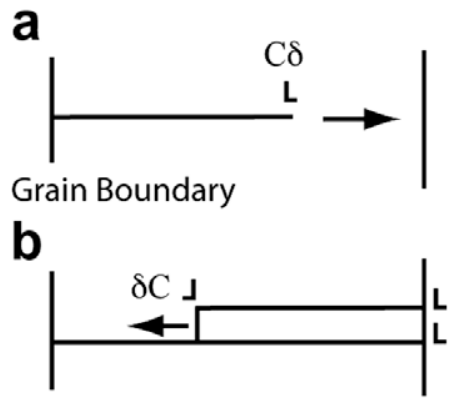

Fig. 7. The formation of a single twin by rebound mechanism. (a) A partial $\mathbf{C} \boldsymbol{\delta}$ glides to the right to form a stacking fault. (b) $\mathbf{C} \boldsymbol{\delta}$ rebounds as $\delta \mathbf{C}$ from a grain boundary or twin boundary to nucleate a single twin. Two partials $\mathbf{C} \boldsymbol{\delta}$ are left superimposed on the grain boundary at the right. 


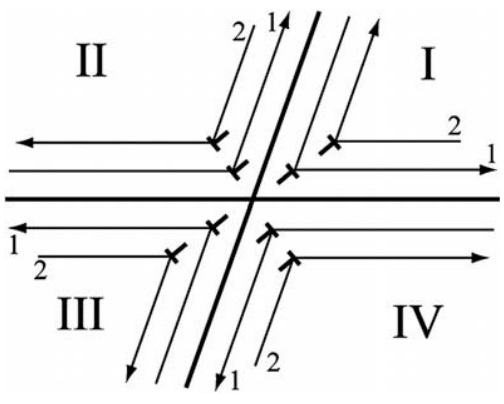

Fig. 8. Schematics of partial reactions in the four reentrant angles in the rebound mechanisms for producing V-, T- and X-shaped multiple twins. 1 represents the first partial slip and 2 represents the slip by the rebound partial. Note that the sequence of slip events is arbitrary, i.e. event 2 could occur before event 1 .

Table 1

The reactions of partial dislocations at the cross-slip points in reentrant angles I-IV.

\begin{tabular}{lllll}
\hline $\begin{array}{l}\text { Reaction } \\
\text { angle I }\end{array}$ & $\begin{array}{l}\text { Reentrant } \\
\text { angle II }\end{array}$ & $\begin{array}{l}\text { Reentrant } \\
\text { angle III }\end{array}$ & $\begin{array}{l}\text { Reentrant } \\
\text { angle IV }\end{array}$ \\
\hline 1 & $\mathbf{C} \boldsymbol{\beta} \rightarrow \mathbf{C} \boldsymbol{\delta}+\boldsymbol{\delta} \boldsymbol{\beta}$ & $\mathbf{C} \boldsymbol{\delta} \rightarrow \mathbf{C} \boldsymbol{\beta}+\boldsymbol{\beta} \boldsymbol{\delta}$ & $\boldsymbol{\beta C} \rightarrow \boldsymbol{\beta} \boldsymbol{\delta}+\boldsymbol{\delta} \mathbf{C}$ & $\boldsymbol{\delta C} \rightarrow \boldsymbol{\delta} \boldsymbol{\beta}+\boldsymbol{\beta C}$ \\
2 & $\boldsymbol{\delta} \mathbf{C} \rightarrow \boldsymbol{\delta} \boldsymbol{\beta}+\boldsymbol{\beta C}$ & $\boldsymbol{\beta C} \rightarrow \boldsymbol{\beta} \boldsymbol{\delta}+\boldsymbol{\delta} \mathbf{C}$ & $\mathbf{C} \boldsymbol{\delta} \rightarrow \mathbf{C} \boldsymbol{\beta}+\boldsymbol{\beta} \boldsymbol{\delta}$ & $\mathbf{C} \boldsymbol{\beta} \rightarrow \mathbf{C} \boldsymbol{\delta}+\boldsymbol{\delta} \boldsymbol{\beta}$
\end{tabular}

These reactions can be easily understood with the assistance of Fig. 2 .

the generation of a full dislocation, a partial and a stair-rod dislocation from one partial. The generation of the full dislocation is required so that it cross-slips into the next slip plane to continue the twin growth. This dislocation reaction has a high-energy barrier, which has to be overcome by a high applied stress.

The rebound mechanism generates the twinning partial on the next slip plane by rebounding the partial impinging on the grain boundary or twin boundary. This process circumvents the need to overcome the high-energy barrier in the self partial-multiplication mechanism. Fig. 8 schematically illustrates how the rebound mechanism operates. Depending on which reentrant angle(s) the rebound mechanism activates, V-, T- and X-shaped multiple twins can be produced. With the help of Figs. 1 and 2, the dislocation reactions at the cross-slip point in each reentrant angle can be determined. They are listed in Table 1. Note that the cross-slip of partials and the dislocation reactions in each reentrant position during the twin growth is almost identical to the "stair-rod cross-slip" mechanism proposed by Fujita and Mori [35].

\section{Experimental evidence of the twinning mechanisms}

Deformation twins with morphologies that are consistent with those of the four types of twins proposed in the previous sections have been observed experimentally. In this section we present transmission electron microscopy (TEM) images in two alloys and high-resolution TEM images of twins observed in nanocrystalline $\mathrm{Al}, \mathrm{Cu}$ and $\mathrm{Ni}$.

We have proposed two different types of mechanism for forming these twins. In the case of single twins, the self par- tial-multiplication mechanism involves a Shockley partial at the twin boundary (see Fig. 3c). This Shockley partial should be associated with, and located at the end of, a stacking fault. Therefore, we should see a twin in the (d) plane and a stacking fault in the (b) plane. Such a configuration is indeed observed in nanocrystalline $\mathrm{Cu}$ processed by high-pressure torsion, as shown in Fig. 9 .

The rebound mechanism can also form a single twin; such a single twin can have the conventional twin morphology, with a macroscopic shear [2]. No image is shown here because these twin morphologies have been reported elsewhere $[2-5,9,10,12,33]$.

The V-shaped double twin that is consistent with the self partial-multiplication mechanism has been reported previously [29]. In the reported twin morphology one of the two twin branches ends in the grain interior, which cannot be formed by the rebound mechanism. This will be discussed in more detail in the Discussion section. The rebound mechanism needs ends of both twin branches to impinge on either a twin boundary or a grain boundary. A V-shaped twin consistent with the rebound mechanism is shown in Fig. 10, in which twins 1 and 2 form a V-shaped

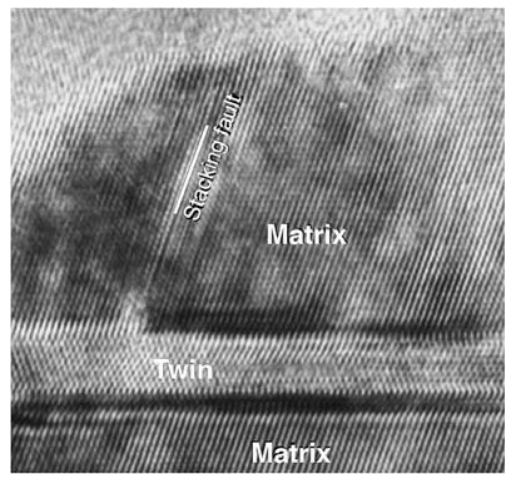

Fig. 9. A single twin that is consistent with the self-generated formation mechanism.

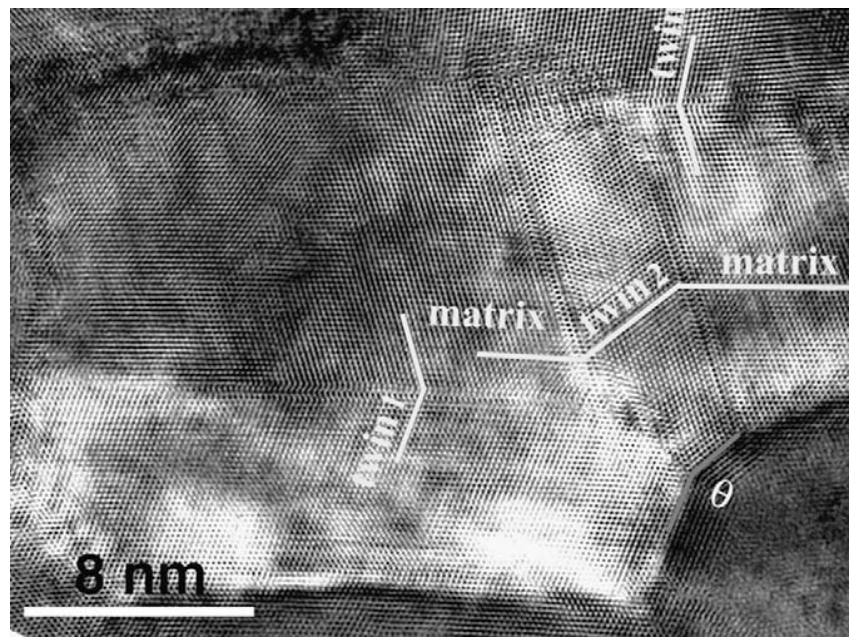

Fig. 10. Twins 1 and 2 form a V-shaped twin that is consistent with the formation mechanism of partial rebound at grain boundaries. 


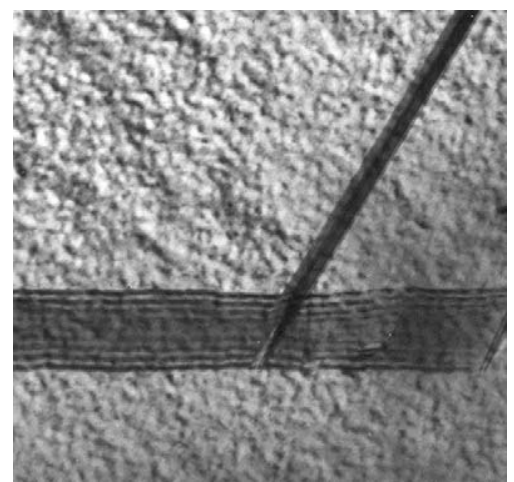

Fig. 11. A T-shaped twin (or stacking fault) framework observed under TEM during in situ deformation of a coarse-grained $\mathrm{Cu}-\mathrm{Ge}(5$ at.\%) alloy. Note that the lines in the micrograph are fringes caused by the inclination of twins or stacking faults with respect to the surface of the TEM foil.

twin. In Fig. 10, only the region where twins 1 and 2 meet each other might be formed by the proposed rebound mechanism for the formation of $\mathrm{V}$-shaped twin. Other parts of the two twins where the slip planes of twin 1 do not meet those from twin 2 could be formed by the rebound mechanism for single twins.

Fig. 11 shows a T-shaped thin twin or stacking fault observed under TEM during in situ deformation in a coarse-grained $\mathrm{Cu}-\mathrm{Ge}(5$ at. $\%)$ alloy with very low SFE. More information can be found in Ref. [28]. Note that the lines in the image are fringe lines because the twin or stacking fault is inclined with respect to the surface of the TEM foil. Fig. 11 demonstrates a framework for growing a T-shaped twin via the mechanisms proposed in this paper. X-shaped twins, where two twin lamellae on the (d) and (b) planes cross each other, have been observed to form readily in AISI 304 stainless steel deformed by surface mechanical attrition treatment (SMAT) [37]. Unfortunately, for the T-shaped and X-shaped twins, only lowmagnification TEM images have been reported. High-resolution TEM investigation is needed to probe the atomic details at the inter-twin boundaries to reveal if they are consistent with the self-source and rebound twinning mechanisms.

\section{Discussion}

We have proposed two types of twinning mechanism the self partial-multiplication mechanism and the rebound mechanism - for the nucleation and growth of four different types of twins: single twins, V-shaped double twins, Tshaped multiple twins and X-shaped multiple twins. Both types of mechanism can cause growth of the twins in a continuous way, and they produce similar final twin morphologies. However, there are differences between these two types of twinning mechanism that may make one mechanism favorable over the other under certain deformation conditions. Below we discuss the features of these two types of twinning mechanism and the deformation conditions that promote their activation.
The self partial-multiplication mechanism produces twinning partials by reactions of dislocations at a twin boundary in the case of the single twin and at the twin-twin boundary in the case of the multiple twins. The dislocation reaction for the formation of a single twin involves a Shockley partial dissociating into a stair-rod dislocation and another Shockley partial on the cross-slip plane. This reaction has a relatively low energy barrier and therefore should not require a very high local driving stress. In contrast, dislocation reaction at the twin-twin boundary for the nucleation and growth of V-, T- and X-shaped multiple twins involves a Shockley partial dislocation dissociating into a stair-rod dislocation, another Shockley partial on the cross-slip plane and a full dislocation. This dislocation reaction has a high-energy barrier and might be difficult to activate. Nanocrystalline materials have a much higher strength than their coarse-grained counterparts [38-40], and therefore deform under very high stress. This high stress helps overcome the high-energy barrier for the dislocation reactions. Therefore, these $\mathrm{V}-, \mathrm{T}$ - and $\mathrm{X}$-shaped twins might form more readily via the self partial-multiplication mechanisms in nanocrystalline fcc metals than in coarse-grained ones. Other deformation conditions, such as low temperature and high strain rate, should also help with the activation of this twinning mechanism because a higher flow stress is produced by these conditions.

The nucleation and growth of multiple twins by the self partial-multiplication twinning mechanism involves the generation of stair-rod dislocations. These stair-rod dislocations have been observed extensively by molecular
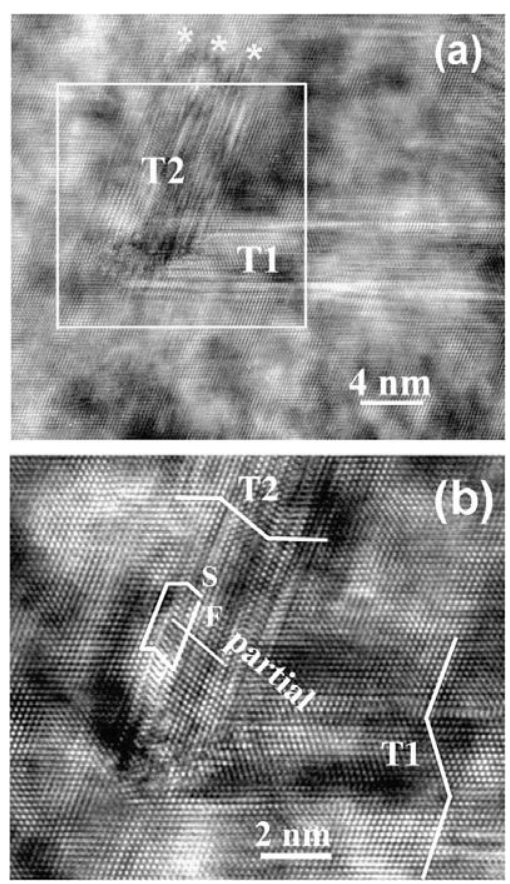

Fig. 12. HREM micrographs showing (a) the image of a V-shaped twin with the twin T2 ending inside the grain interior, as marked by asterisks, and (b) the enlarged image of twin-twin boundary area in the white frame in (a). 
dynamics simulations during the deformation of nanocrystalline Al $[41,42]$. Note that the stair-rod dislocation may not form under all of the applied stress conditions; for example, Jin et al. did not observe any during the simulation of nanoindentation of $\mathrm{Al}\left(\begin{array}{lll}0 & 0 & 1\end{array}\right)$ surface [43]. The stair-rod dislocations were also observed experimentally. For example, as shown in Fig. 11, a V-shaped twin consisting of twins $\mathrm{T} 1$ and $\mathrm{T} 2$ was observed in nanocrystalline $\mathrm{Ni}$ [29]. Twin T2 ends inside the grain interior (marked by asterisks in Fig. 12a), which indicates that the partials forming T2 could only be generated from the $\Sigma 9$ twin-twin boundary of $\mathrm{T} 1$ and $\mathrm{T} 2$. The dislocation reactions generating such partials will produce stair-rod dislocations, and the stress field of such a stair-rod dislocation array causes lattice distortion, as seen in Fig. 12b. These molecular dynamics simulation and experimental results indicate that the proposed self partial-multiplication twinning mechanisms can indeed operate in nanocrystalline fcc metals despite requiring a high applied stress.

The rebound mechanisms should be more sensitive to the velocity of the Shockley partial dislocation when it impinges on a grain boundary or twin boundary. High velocity should make the rebound mechanism easier to activate. Therefore, high strain rate deformation might be more favorable to the rebound mechanism. In fact, the extensive X-shaped twins observed in stainless steel were produced at a relatively high strain rate under SMAT [38].

One issue about the V-, T- and X-shaped multiple twins formed by either the self partial-multiplication mechanism or the rebound mechanism is the pileup of stair-rod dislocations on every slip plane. This will result in high local stress at the twin-twin boundaries, although long-range stress might be relieved partially (T-shaped twins) or fully (X-shaped twins).

\section{Summary}

We have proposed two types of twinning mechanism for the formation of single twins and V-, T- and X-shaped multiple twins. Both types of mechanism provide a way for the twin to grow/thicken by a continued generation of twinning partials on the adjacent slip planes. The first type is named the self partial-multiplication twinning mechanism because the twinning partial is generated at the twin boundaries or at the intersection of twin boundaries. Activation of this type of mechanism requires a high stress. The other type is called the rebound mechanism because the twinning partial is generated by the reflection of the stress field of the Shockley partial impinging at a grain boundary or twin boundary. A high gliding speed of the partial (or a high strain rate) should promote this type of twinning mechanism. More experimental study is needed to further verify these twinning mechanisms.

\section{References}

[1] Christian JW, Mahajan S. Prog Mater Sci 1995;39:1.

[2] Wu XL, Liao XZ, Srinivasan SG, Zhou F, Lavernia EJ, Valiev RZ, et al. Phys Rev Lett 2008;100:095701.

[3] Chen MW, Ma E, Hemker KJ, Sheng HW, Wang YM, Cheng XM. Science 2003;300:1275.

[4] Liao XZ, Zhao YH, Zhu YT, Valiev RZ, Gunderov DV. J Appl Phys 2004;96:636.

[5] Liao XZ, Zhao YH, Srinivasan SG, Zhu YT, Valiev RZ, Gunderov DV. Appl Phys Lett 2004;84:592.

[6] Yamakov V, Wolf D, Phillpot SR, Mukherjee AK, Gleiter H. Nat Mater 2002;1:45

[7] Van Swygenhoven H, Derlet PM, Froseth AG. Nat Mater 2004;3:399.

[8] Meyers MA, Vohringer O, Lubarda VA. Acta Mater 2001;49:4025.

[9] Wu XL, Zhu YT. Phys Rev Lett 2008;101:025503.

[10] Liao XZ, Zhou F, Lavernia EJ, Srinivasan SG, Baskes MI, He DW, et al. Appl Phys Lett 2003;83:632.

[11] Yamakov V, Wolf D, Phillpot SR, Mukherjee AK, Gleiter H. Nat Mater 2004:3:43

[12] Wang J, Huang HC. Appl Phys Lett 2004;85:5983.

[13] Wu XL, Zhu YT. Appl Phys Lett 2006;89:031922.

[14] Liao XZ, Zhou F, Lavernia EJ, He DW, Zhu YT. Appl Phys Lett 2003;83:5062.

[15] Yamakov V, Wolf D, Phillpot SR, Gleiter H. Acta Mater 2002;50:5005.

[16] Zhu YT, Liao XZ, Zhao YH, Srinivasan SG, Zhou F, Lavernia EJ. Appl Phys Lett 2004;85:5049.

[17] Zhu YT, Liao XZ, Srinivasan SG, Lavernia EJ. J Appl Phys 2005;98:034319.

[18] Liao XZ, Huang JY, Zhu YT, Fhou F, Lavernia EJ. Philos Mag 2003;83:3065.

[19] Zhu YT, Liao XZ, Valiev RZ. Appl Phys Lett 2005;86:103112.

[20] Huang JY, Wu YK, Ye HQ. Acta Mater 1996;44:1121.

[21] Cao AJ, Wei YG. Appl Phys Lett 2006;89:041919.

[22] Ookawa A. J Phys Soc Jpn 1957;25:825.

[23] Venables JA. Philos Mag A 1961;6:379.

[24] Niewczas M, Saada G. Philos Mag A 2002;82:161.

[25] Mahajan S, Chin GY. Acta Metall 1973;21:1353.

[26] Mahajan S, Green ML, Brasen D. Metall Trans A 1977;8:283.

[27] Thompson N. Proc Phys Soc London Sect B 1953;66:481.

[28] Narayan J, Zhu YT. Appl Phys Lett 2008;92:151908.

[29] Wu XL, Narayan J, Zhu YT. Appl Phys Lett 2008;93:031910.

[30] Dregia SA, Hirth JP. J Appl Phys 1991;69:2169.

[31] Henager Jr CH, Hoagland RG. Scripta Mater 2004;50:701.

[32] Narayan J, Oktyabrshy S. J Appl Phys 2002;92:7122.

[33] Rösner H, Markmann J, Weissmüller J. Philos Mag 2004;84:321.

[34] Hirth JP, Lothe J. Theory of dislocations. 2nd ed. Malabar (FL): Krieger; 1992.

[35] Fujita H, Mori T. Scripta Metall 1975;9:631.

[36] Akarapu S, Zbib H, Hirth JP. Scripta Mater 2008;59:256002E.

[37] Zhang HW, Hei ZK, Liu G, Lu J, Lu K. Acta Mater 2003;51:1871.

[38] Zhu YT, Liao XZ. Nat Mater 2004;3:351.

[39] Youssef KM, Scattergood RO, Murty KL, Horton JA, Koch CC. Appl Phys Lett 2005;87:091904.

[40] Weertman JR. Mater Sci Eng 1993;166:161.

[41] Yamakov V, Wolf D, Phillpot SR, Gleiter H. Acta Mater 2003;51:4135.

[42] Wolf D, Yamakov V, Phillpot SR, Mukherjee A, Gleiter H. Acta Mater 2005;53:1.

[43] Jin J, Shevlin SA, Guo ZX. Acta Mater 2008;56:4358. 